\title{
Prevención de la infección nosocomial en pacientes críticos
}

\section{Prevention of nosocomial infection in critical patients}

\author{
M. Palomar ${ }^{a}, *$, P. Rodríguez ${ }^{\mathrm{b}}$, M. Nieto $^{\mathrm{c}}$ y S. Sancho ${ }^{\mathrm{d}}$ \\ aServicio de Medicina Intensiva, Hospital Universitario Vall d'Hebrón, Barcelona, España \\ ${ }^{\mathrm{b} S e r v i c i o}$ de Medicina Intensiva, Hospital Universitario La Fe, Valencia, España \\ 'Servicio de Medicina Intensiva, Hospital Universitario San Carlos, Madrid, España \\ ${ }^{\mathrm{d}}$ Servicio de Medicina Intensiva, Hospital Universitario Dr. Peset, Valencia, España
}

Recibido el 12 de enero de 2010; aceptado el 12 de marzo de 2010

Disponible en Internet el 26 de mayo de 2010

\section{Introducción}

Las infecciones asociadas a cuidados sanitarios son errores prevenibles en su mayoría. Según las estimaciones del Institute of Medicine, hasta 100.000 pacientes podrían fallecer debido a errores médicos ${ }^{1}$. A excepción de urgencias, pocos servicios son tan complejos como las unidades que atienden a pacientes críticos, y la posibilidad de que durante la atención sanitaria se produzcan incidentes, errores, complicaciones o como queramos denominarlos, se incrementa proporcionalmente a la complejidad del medio. En 1995, un estudio determinó que en las Unidades de Cuidados Intensivos ( $\mathrm{UCl}$ ) de hospitales universitarios americanos, anualmente se producen hasta 150.000 errores graves con deterioro vital y que, diariamente, un paciente está expuesto a 1,7 incidentes ${ }^{2}$. En un reciente estudio realizado en las $\mathrm{UCl}$ de nuestro país se detectaron 1,22 incidentes por cada paciente ingresado y 5,89 incidentes por 100 pacientes y hora ${ }^{3}$.

Los sistemas de notificación muestran que las infecciones son errores clave tanto por su número como por su impacto ${ }^{4}$, y las que desarrollan los pacientes críticos suponen hasta el 20-25\% del total de infecciones nosocomiales (IN). El hecho de que los pacientes críticos presenten la mayor suscepti-

*Autor para correspondencia.

Correo electrónico: mpalomar@telefonica.net (M. Palomar). bilidad, sumada a la frecuente alteración de sus barreras defensivas por dispositivos invasivos, en un ambiente con numerosas oportunidades de transmisión cruzada y con un ecosistema seleccionado han justificado durante mucho tiempo esas cifras. Pero el concepto de «inevitabilidad» de las IN-UCl (como también de otros incidentes) ha dejado paso a la convicción y al conocimiento de que la gran mayoría (no todas) son prevenibles y que no es aceptable no poner todos los medios posibles para reducir su impacto.

Este mensaje matiza el concepto que está implantándose en áreas o sistemas sanitarios de algunos países de que el riesgo puede ser 0 si la tolerancia lo es, hasta el punto de que en EE. UU. el Medicare no abona a los hospitales algunas de las $\mathbb{I N}^{5,6}$, o se potencia la posibilidad de compensaciones económicas. Algunos autores ${ }^{7-9}$ han alertado de que este nuevo escenario crea falsas expectativas, que podrían estimular la tentación de no declarar las infecciones reales, y así falsear la realidad, lo que no debería extrañarnos, ya que uno de los requisitos para que los sistemas de notificación tengan éxito es que no sean punibles ${ }^{3}$.

La prevención de las $\mathbb{I N}$ incluye medidas generales comunes para todas las infecciones y otras específicas para cada localización, que se basan en la fisiopatología de cada infección y ambas vertientes deben implementarse a través de la educación, la disminución de los factores de riesgo y la adherencia a guías.

En esta revisión abordaremos las estrategias preventivas con este esquema, incluidas medidas específicas tan solo 
para las 2 IN con mayor impacto en UCl: la neumonía asociada a ventilación mecánica (NAV) y la bacteriemia relacionada con catéter (BRC).

\section{Medidas generales}

\section{Estrategias para evitar la transmisión horizontal}

Las infecciones exógenas tienen su origen tanto en reservorios inanimados (respiradores, sistemas de monitorización, superficies, etc.) como en portadores (trabajadores sanitarios o pacientes colonizados-infectados) y pueden transmitirse durante la práctica de la higiene o en el tratamiento terapéutico de los pacientes. Las estrategias para evitar la transmisión horizontal son comunes a todas las infecciones e incluyen las intervenciones que se muestran en la tabla 1 y que ahora revisaremos.

Higiene de manos. A pesar de ser un concepto que viene del siglo XIX, su cumplimiento sigue siendo un reto en la actualidad. Ha sido el primer objetivo escogido por la Organización Mundial de la Salud en el lanzamiento de la alianza por la seguridad del paciente ${ }^{10}$. El concepto «Cuidado limpio es cuidado seguro» es especialmente relevante en las $\mathrm{UCl}$, ya que cada hora se tienen hasta 30 oportunidades para desinfectar las manos, lo que requiere un tiempo que compite con el de la asistencia. La incorporación de las soluciones alcohólicas ha permitido reducir el tiempo necesario para realizar la higiene y de ese modo acercarse a un grado de cumplimiento más aceptable que los observados con el lavado tradicional.

Limpieza ambiental. Fallos en la limpieza de las habitaciones se han asociado con la adquisición en UCl de Staphilococcus aureus resistente a meticilina (SARM), Acinetobacter baumannii o enterococo resistente a vancomicina $^{10,11}$, por lo que actualmente se pone un gran énfasis en la limpieza de superficies y objetos ambientales, sobre todo antes del ingreso de los pacientes ${ }^{12}$.

Aislamiento de contacto. Indicado en pacientes infectados o colonizados por microorganismos resistentes. Las medidas incluyen distintas posibilidades, uso de precauciones de barrera (guantes, batas, mascarillas), habitaciones de

Tabla 1 Medidas generales de prevención de infección nosocomial en unidad de cuidados intensivos

1. Estrategias para evitar la transmisión horizontal Higiene de manos

Higiene ambiental

Detección de portadores de bacterias resistentes

Aislamiento

Evitar ratios enfermera/paciente insuficientes

2. Estrategias funcionales

Vigilancia de la infección nosocomial y benchmarking

Programas educacionales

Reducción de la exposición a dispositivos invasivos

Reducción de la estancia en UCI

Seguimiento de bundles

Programas de seguridad

UCl: unidad de cuidados intensivos. aislamiento (individuales o no) o cohortización de la enfermería. Añadir métodos para la detección rápida de los pacientes que ingresan con riesgo de ser portadores, junto con el aislamiento preventivo, parece potenciar la reducción de la transmisión. El ejemplo más conocido es la política de search and destroy seguida en Holanda, que ha hecho desaparecer prácticamente el SARM de su medio ${ }^{13}$.

Evitar sobrecarga de trabajo. Especialmente del personal de enfermería, que se ha asociado a incremento de IN y adquisición de $\mathrm{SARM}^{14,15}$. Si a la sobrecarga se añade hacinamiento, se incrementan los contactos entre sanitarios y diferentes pacientes, por lo que se multiplican las posibilidades de transmisión cruzada de bacterias resistentes, los requerimientos de higiene de manos, la recogida de muestras para detectar portadores, es decir, mayor carga de trabajo, mayores riesgos de error e incluso de burnout ${ }^{16}$.

\section{Estrategias funcionales}

Vigilancia de las infecciones nosocomiales y benchmarking. Ambas actividades contribuyen al control ${ }^{17}$. El primer requisito para combatir un problema es conocer su presencia y magnitud. Pero si la información no se transmite al personal implicado y no se establecen medidas correctoras, la vigilancia es inútil. El impacto del programa SENIC en EE. UU. demostró hace más de 30 años una reducción global del $33 \%$ de las IN en los hospitales participantes, que fue más importante para las bacteriemias e infecciones quirúrgicas que para las neumonías. Por el contrario, los hospitales no adscritos al SENIC vieron incrementadas sus tasas en un $17 \%$ en el mismo período. En nuestro país, las tasas que muestra el estudio ENVIN-HELICS son, por lo general, notablemente inferiores en las $\mathrm{UCl}$ con más antigüedad en la vigilancia o que la realizan de forma continuada durante todo el año.

Formación del personal sanitario. Es un factor fundamental, ya que la inexperiencia se asocia a una elevación de los efectos adversos, incluidas las IN. Es clásico el incremento de las tasas de infección o la aparición de brotes durante los períodos vacacionales, en los que además de que las ratios de personal se pueden ver alteradas, la inexperiencia profesional es más frecuente. La formación específica de los sanitarios implicados en el tratamiento de pacientes mediante programas educativos se ha asociado a excelentes resultados en la prevención de IN, y son muy destacados los resultados de los dedicados a prevenir las $\mathrm{BRC}^{18,19}$.

Protocolos. Además de los protocolos específicos de prevención de IN en las distintas localizaciones, y dado que la exposición a los factores de riesgo es una de las principales causas facilitadoras de infección, la reducción de la estancia en UCI a través de la implementación de protocolos específicos de sedación, destete, nutrición, etc., que limiten la exposición a estos factores y reduzcan la estancia en $\mathrm{UCl}$ se ha mostrado eficaz en la reducción de las tasas de infección 20,21.

Bundles. En los últimos años, en la elaboración de protocolos se ha incorporado un concepto originario del marketing, el uso de bundles, es decir, la aplicación simultánea de una serie de medidas basadas en la evidencia, que en su aplicación conjunta mejoran sustancialmente el 
resultado de los pacientes, lo que asegura que no dejan de recibir el mejor tratamiento posible $e^{22}$.

$\mathrm{El} \mathrm{IHI} \mathrm{es} \mathrm{uno} \mathrm{de} \mathrm{los} \mathrm{principales} \mathrm{promotores} \mathrm{del} \mathrm{concepto}$ bundles, y una de las más difundidas es la referida a los pacientes con ventilación mecánica $(\mathrm{VM})^{23}$, que consiste en 4 medidas: elevación del cabecero, interrupción diaria de la sedación con valoración de la posibilidad de extubación y las profilaxis de la úlcera péptica y la trombosis venosa. Como podemos ver, solo 2 de los 4 componentes son específicos para la prevención de la NAV, mientras los otros previenen otras complicaciones.

Programas de seguridad. Los principios para la seguridad del paciente y de mejoría de la calidad se han empezado a incorporar a los programas de prevención de la $I^{24}$. Entre los pioneros, está la campaña «Salvar 100.000 vidas» promovida por el IHI, que tenía como uno de los objetivos principales en los pacientes críticos la prevención de la $\mathrm{NAV}^{25}$. El concepto es aplicable a todas las infecciones y quizás el ejemplo más exitoso corresponde a la iniciativa de Peter Pronovost en Michigan en relación con la $\mathrm{BRC}^{26}$.

Entre los componentes de estos programas se encuentran, además de la formación, intervenciones dirigidas a mejorar la comunicación entre estamentos y reordenar el trabajo para que se realice en equipo mediante el uso de listas de comprobación, monitorización de procedimientos e indicadores. La estrategia para lograrlo se componía de 4 puntos: implicar al personal sanitario, educar en la evidencia, ejecutar el programa y evaluar los resultados. El responsable de su implantación era un equipo líder compuesto por un médico y una enfermera de la unidad, pero también el personal directivo estaba implicado estrechamente en el proceso de cambio. Aunque el análisis del resultado se centraba en la reducción de la tasa de bacteriemia, el objetivo central era mejorar la seguridad de los pacientes a partir de ese objetivo concreto.

\section{Bacteriemia relacionada con catéter}

La BRC puede originarse a partir de 3 vías patogénicas ${ }^{27}$ : 1) la llamada vía exoluminal o el progreso de la flora cutánea a partir del punto de entrada, que coloniza el segmento subcutáneo y posteriormente el resto del catéter; 2) la vía endoluminal, en la que la colonización del catéter se produce a través de las manipulaciones de la conexión o de los puertos sin aguja, y 3) la menos frecuente, la vía hematógena o colonización del catéter a partir de otros focos distales de infección.

La vía exoluminal es más frecuente en la primera semana tras la inserción del catéter y está muy relacionada con el proceso de la inserción. Algunas localizaciones como la femoral o yugular se acompañan de infección con más frecuencia que cuando la inserción es en el antebrazo o en la subclavia ${ }^{28,29}$. En contraste, la vía endoluminal es independiente de la localización pero está relacionada con la higiene y el número de manipulaciones de la conexión. La BRC de este origen suele ser más tardía. La etiología de las BRC tempranas corresponde mayoritariamente a grampositivos (GP), mientras que las BRC tardías o con origen en catéteres femorales se acompañan de infección por bacilos gramnegativos (BGN) y hongos en mayor proporción ${ }^{30}$.
Las medidas preventivas pueden incluir numerosos aspectos que están recogidos en diferentes guías y recomendaciones de agencias sanitarias, sociedades científicas, comunidades autónomas (CC. AA.), comisiones de infecciones de los hospitales, etc., y se agrupan en 2 bloques: las medidas para el momento de la inserción y las relativas al mantenimiento del catéter ${ }^{27,31,32}$. Sin embargo, la experiencia de Michigan apoya seleccionar entre las decenas de medidas propuestas la aplicación de una bundle sencilla que incluye tan solo las 5 medidas con mayor evidencia y más facilidad para implementarse complementada con un programa de seguridad.

Las medidas seleccionadas, todas ellas con fuerza máxima de recomendación, fueron las siguientes: 1) higiene de manos antes de la inserción y manipulación de los catéteres; 2) desinfección de la piel con clorhexidina; 3) medidas de barrera máxima durante la inserción de los catéteres; 4) evitar la localización femoral, y 5) retirada de los catéteres innecesarios.

La estrategia de seguridad complementaria para asegurar el cumplimiento consistió en formar a los clínicos en las prácticas de prevención y en el conocimiento de los daños derivados de la adquisición de BRC; crear unos carros para la inserción, que contengan todo el material necesario para el procedimiento y así facilitar el cumplimiento de las buenas prácticas; utilización de una lista de comprobación durante la inserción que asegure la correcta práctica y otorgue poder a la enfermería (en situaciones no urgentes) para interrumpir el procedimiento si no se seguían las normas; diariamente, durante las visitas conjuntas, valoración de la posibilidad de retirar los catéteres o reducir su manipulación, $y$, finalmente, información mensual de las tasas de BRC, con discusión de los episodios diagnosticados, análisis de por qué se habían desarrollado y si podían haberse evitado. Finalmente, se establecían objetivos de mejora para los meses siguientes.

\section{Bacteriemia Zero}

A pesar de los numerosos protocolos, consensos y recomendaciones de hospitales o sociedades científicas, las tasas en España han permanecido en cifras superiores a las de otros países europeos vecinos o a las publicadas en los estudios de vigilancia americanos ${ }^{33,34}$. Para intentar modificar esta situación, la experiencia americana se ha trasladado a España gracias a un acuerdo de SEMICYUC con la Agencia de Calidad del Ministerio de Sanidad, Consumo y Política Social en colaboración con la Alianza Mundial por la Seguridad del Paciente de la Organización Mundial de la Salud. Tras un estudio piloto realizado durante el último trimestre de 2007, que mostró la viabilidad del proyecto (reducción de la tasa en un 50\%) y las dificultades para su implantación, en 2009 todas las CC. AA. se han adherido al nuevo proyecto denominado Bacteriemia Zero.

El objetivo principal es reducir la media estatal de la DI de la BRC a menos de 4 episodios de bacteriemia por 1.000 días de Catéter Venoso Central (CVC) (reducción del 40\% respecto a la tasa media de los últimos 5 en las $\mathrm{UCl}$ españolas). Como objetivos secundarios se encuentran: 1) promover y reforzar la cultura de seguridad en las $\mathrm{UCI}$ del SNS; 2 ) crear una red de $\mathrm{UCl}$, a través de las CC. AA., que 
Tabla 2 Contenido del proyecto Bacteriemia-Zero

STOP-Bacteriemia

a. Higiene adecuada de manos

b. Uso de clorhexidina en la preparación de la piel

c. Uso de medidas de barrera total durante la inserción de los CVC

d. Preferencia de la vena subclavia como lugar de inserción

e. Retirada de CVC innecesarios

f. Tratamiento higiénico de los catéteres

Plan de seguridad integral

a. Evaluar la cultura de seguridad (medición basal y periódica

b. Formación en seguridad del paciente

c. Identificar errores en la práctica habitual (por los profesionales)

d. Establecer alianzas con la dirección de la institución para la mejora de la seguridad. Aprender de los errores

CVC: catéter venoso central.

apliquen prácticas seguras de efectividad demostrada, y 3) documentar todos los episodios de bacteriemia, incluidas las secundarias de otros orígenes, así como la etiología de estas y las características de los pacientes que las desarrollan.

El proyecto Bacteriemia Zero se estructura en 2 vertientes: 1) STOP-BRC, que incluye medidas específicas y estandarizadas relacionadas con la inserción y el manejo de los CVC, y 2) un plan de seguridad integral dirigido a promover la cultura de seguridad en el trabajo diario (tabla 2).

La STOP-BRC incluye las medidas del estudio de Michigan con 2 modificaciones: la primera, promover el uso de la localización en la subclavia, en caso de no poder utilizar un catéter central de inserción periférica; la segunda diferencia es insistir en la recomendación de manejar el catéter de forma higiénica, dado que en nuestro medio la duración media hasta el desarrollo de bacteriemia es de 2 semanas tras el ingreso en $\mathrm{UCl}$ y la vía endoluminal tiene tanta o más importancia que la exoluminal.

Higiene de manos. En contra de lo esperable, esta actividad sigue presentando un bajo nivel de cumplimiento, especialmente entre el personal médico. Es imprescindible tanto durante la inserción como en la manipulación de los catéteres, para evitar la colonización de la piel, el propio catéter o las conexiones ${ }^{31}$. Las oportunidades de practicar la higiene de manos incluyen situaciones que no siempre se tienen presentes, como antes y después del uso de guantes, entre pacientes o tras ir al baño. La monitorización de la higiene de manos forma parte de la lista de comprobación durante la inserción del catéter.

Uso de barreras máximas durante la inserción. Las medidas de esterilidad máximas, incluido el uso de guantes, bata, gorro y paños que cubran totalmente al paciente frente a unas medidas mínimas o menos exigentes, se ha demostrado, fundamentalmente por el estudio de Raad y apoyado por el de Mermel et $\mathrm{al}^{35,36}$, que son significativamente útiles en la reducción de BRC y costeefectivas, ya que por cada 270 catéteres implantados con máxima esterilidad se evitan 7 BRC $^{35}$.
Desinfección de la piel con clorhexidina. Hasta ahora, el desinfectante más usado en la inserción o mantenimiento de los catéteres había sido la povidona yodada; sin embargo, la evidencia tanto en estudios individuales como en metaanálisis apoya fuertemente su sustitución por clorhexidina $^{37}$. La transparencia ha hecho que algunos sanitarios la rechacen, por lo que la opción de usar clorhexidina tintada se está implantando en nuestro país.

Potenciar el uso de la localización en la subclavia. Tanto la localización femoral como la yugular presentan tasas más elevadas de BRC que en el caso de la subclavia y, por supuesto, que en las vías centrales de inserción periférica. Se recomienda la elección de subclavia si no existen contraindicaciones médicas ${ }^{31,32}$.

Retirar los catéteres innecesarios. La exposición al dispositivo es el principal factor de riesgo para desarrollar infección. Si en el momento de la inserción debe escogerse el CVC con menor número de luces que posibilite la atención al paciente, diariamente debe plantearse si el/ los CVC, o todas las luces utilizadas siguen siendo necesarios o si otras rutas de administración son posibles ${ }^{31}$. La sustitución sistemática de los CVC no previene la infección, y el recambio mediante guía metálica incrementa el riesgo de $\mathrm{BRC}^{38}$.

Manejo higiénico de los catéteres. Además de la higiene de manos previa a cualquier manejo de los catéteres, la desinfección de las conexiones o de los dispositivos sin agujas con una solución de gluconato de clorhexidina al $2 \%$ o alcohol de $70^{\circ}$ antes y después del acceso a estos reduce el riesgo de colonización-infección endoluminal. Los dispositivos sin agujas, creados para reducir el riesgo de pinchazos de los sanitarios pueden, además, reducir la tasa de $\mathrm{BRC}^{39,40}$ si se utilizan correctamente, pero si no se manejan higiénicamente, siguen protegiendo al personal pero incrementan el riesgo de infección del CVC $^{41}$.

La protección del punto de inserción con apósitos adecuados minimiza la infección. Recientemente, un estudio multicéntrico francés ha demostrado que incluso las tasas bajas de bacteriemia pueden reducirse mediante la aplicación de cobertura impregnada en clorhexidina ${ }^{42}$. El mismo estudio mostró que es seguro dilatar hasta 7 días el cambio de los equipos de infusión, a menos que esté clínicamente indicado. Esa norma cambia (aunque con menor grado de recomendación) cuando los equipos se utilizan para administrar sangre, productos sanguíneos o lípidos, y pasan al cambio dentro de las $24 \mathrm{~h}$ tras el inicio de la perfusión.

El uso de CVC impregnados en antibióticos/antisépticos solo se recomienda en centros en los que se mantienen tasas elevadas a pesar de seguir las medidas básicas de mayor evidencia descritas previamente o en pacientes con limitación de accesos ${ }^{31,43,44}$. La razón es que tan solo los metaanálisis han logrado demostrar disminución del riesgo de BRC al utilizar catéteres impregnados en clorhexidina-sulfadiazina argentica o en rifampicina-minociclina, y en la mayoría de los estudios el acceso femoral se empleó poco. Por otra parte, su efecto radica fundamentalmente sobre GP y poco sobre BGN u hongos (más frecuentes en localización femoral), es decir, disminuye el riesgo de infección por parte de los gérmenes con menor impacto. 


\section{Neumonía asociada a ventilación mecánica}

La NAV es una de las IN más prevalentes y graves del paciente crítico $^{45}$. La patogenia de la NAV tiene una estrecha relación con la presencia del tubo endotraqueal (TET), hasta tal punto que algunos autores han sugerido un cambio nominal a neumonía asociada al TET. La inserción del TET es una maniobra agresiva que con frecuencia produce una lesión de la mucosa traqueal e implanta un inóculo bacteriano exógeno o endógeno. El TET elimina algunos de los mecanismos naturales de defensa de la vía aérea y vehiculiza los microorganismos al interior del pulmón mediante la entrada de las secreciones subglóticas o la formación del biofilm en la superficie del TET.

La primera medida preventiva es el cese de la VM en cuanto esta no sea necesaria. En segundo lugar, para evitar la infección exógena deben aplicarse estrictamente las medidas habituales sobre la higiene de manos y la asepsia en la manipulación del TET. Una vez comprobado que la VM es inevitable y que la política general de control de la IN se aplica correctamente, los esfuerzos deben dirigirse a evitar las 2 principales vías patogénicas de la NAV: el paso a la vía aérea de las secreciones del espacio subglótico y la formación de biofilm sobre el TET.

Otras estrategias dirigidas a otros aspectos, como el control estricto de la glucemia, el uso de protectores de la mucosa gástrica o la administración de hemoderivados, han mostrado, en ocasiones, cambios en la incidencia de NAV pero parecen prevalecer en su indicación otros efectos sobre la evolución del enfermo ${ }^{45}$.

\section{Secreciones del espacio subglótico}

La acumulación en el espacio subglótico de secreciones procedentes de la orofaringe o del tracto gastrointestinal es un hecho constatado mediante la realización de radiografías $^{46}$ o la cuantificación del material obtenido por aspiración local ${ }^{47}$. La colonización, endógena o exógena, de estas secreciones es un hecho prácticamente inevitable, y la relación causal con la NAV está bien establecida ${ }^{47-51}$. Las medidas preventivas destinadas a evitar la NAV incluyen disminuir la cantidad de secreciones, impedir el paso entre el tubo y la pared traqueal, aspirar las secreciones o esterilizarlas.

Postura del enfermo intubado. La posición supina favorece el reflujo gatroesofágico y, por tanto, un aumento de la cantidad de secreciones acumuladas en el espacio subglótico $^{49}$. Se ha demostrado que la posición semiincorporada del paciente ventilado disminuye la incidencia de NAV (el $23 \%$ en posición supina y el $5 \%$ en posición semiincorporada; $p=0,018)$. Este efecto es mucho más evidente cuando se nutre al paciente por vía enteral ${ }^{52}$. Sin embargo, algunos autores ${ }^{53}$ han encontrado dificultades para mantener la posición a $45^{\circ}$ de forma continuada en todos los pacientes críticos ${ }^{50}$.

Control de neumotaponamiento. El mantenimiento de una presión de neumotaponamiento $\left(\mathrm{P}_{\text {cuff }}\right)$ correcta es imprescindible en los pacientes que reciben $\mathrm{VM}^{48,54,55}$. Una presión excesiva puede alterar la microcirculación de la mucosa traqueal y causar lesiones isquémicas ${ }^{56,57}$, y una $\mathrm{P}_{\text {cuff }}$ insuficiente impide una ventilación con presión positiva y puede permitir la entrada de secreciones subglóticas entre el tubo y la tráquea. Rello et al analizaron el efecto que el control del neumotaponamiento tenía sobre el desarrollo de $\mathrm{NAV}$ en los 8 primeros días de VM y demostraron que una $\mathrm{P}_{\text {cuff }}$ superior a $20 \mathrm{~cm} \mathrm{H}_{2} \mathrm{O}$ de forma mantenida se asociaba a un menor riesgo. Sin embargo, el análisis multivariante sólo pudo demostrar que la $\mathrm{P}_{\text {cuff }}$ inferior a $20 \mathrm{~cm} \mathrm{H} \mathrm{H}_{2} \mathrm{O}$ de forma mantenida era un factor de riesgo independiente en el subgrupo de pacientes sin tratamiento antibiótico ${ }^{58}$.

Varios trabajos constatan el paso de secreciones a pesar de una $P_{\text {cuff }}$ correcta $e$ incluso superior a los límites habituales. Este fenómeno se ha explicado por la formación de pliegues en el neumotaponamiento que permitirían la entrada longitudinal ${ }^{59-61}$. La eliminación de los pliegues se ha logrado mediante el uso de geles lubricantes (eficaz durante $24-120 \mathrm{~h})^{62}$, neumotaponamientos de silicona ${ }^{63}$ o el uso de neumotaponamientos de bajo volumen ${ }^{64}$, pero no se ha comprobado si estas mejoras se acompañan de una menor incidencia de NAV. Más recientemente, en un estudio prospectivo y aleatorizado, Lorente et al comprobaron una clara reducción de la NAV (el 7,9 versus el $22 \%$; $p=0,001$ ) mediante el uso de TET en los que se combinaba un neumotaponamiento de poliuretano y un mecanismo para la aspiración continua de las secreciones subglóticas ${ }^{65}$.

Aspiración de las secreciones subglóticas. Sobre la base de la ineficacia del neumotaponamiento para evitar la aspiración y del considerable volumen de secreciones que se acumulan en el espacio subglótico ${ }^{46}$, se desarrolló un nuevo TET (Hi-Lo Evac tube, Mallinckrodt ${ }^{\circledR}$ ) que dispone de una luz dorsal independiente por la que es posible aspirar las secreciones subglóticas.

Existen 5 estudios prospectivos y aleatorizados que evalúan el efecto de la aspiración intermitente o continua de las secreciones subglóticas ${ }^{47,50,51,67,68}$. En todos los trabajos se logra disminuir el número de episodios de NAV, evaluado bien en forma de tasa de incidencia o de densidad de incidencia (tabla 3).

Con respecto a la influencia que la aspiración de las secreciones subglóticas (ASS) tiene sobre el momento de aparición de la NAV, 3 trabajos coinciden en que produce un retraso en el desarrollo de NAV (todos con significación estadística $)^{47,51,67}$. No parece existir efecto sobre la mortalidad, la duración de la VM o la estancia en la $\mathrm{UCl}$ y en el hospital ${ }^{50,51,67}$, excepto en el caso del trabajo de Bouza et al en el que se logra una disminución significativa de la estancia en $\mathrm{UCl}^{68}$.

La ASS no afecta del mismo modo a todos los microorganismos involucrados en la colonización/infección pulmonar. El efecto protector descrito por Valles y Smulders ${ }^{50,51,67}$ se deriva de una disminución del número de neumonías causadas por gérmenes GP. El diferente efecto sobre los microorganismos podría ser secundario a la selección de NAV tardías o podría deberse, al menos parcialmente, a una acción directa sobre la etiología. La ASS disminuye la cantidad de microorganismos que alcanzan el árbol bronquial y, dado que el inóculo necesario para causar una NAV por cocos GP es mucho mayor que el necesario en el caso de Enterobacteriaceae o Pseudomonas aeruginosa $a^{68,69}$, este efecto cuantitativo podría explicar las diferencias. Otra posible explicación se basa en que no todos los microorganismos siguen el mismo patrón de colonización de las vías aéreas ${ }^{70-73}$. El $P$. aeruginosa, y probablemente otros $B G N$ no fermentadores, parecen tener 
Tabla 3 Estudios prospectivos y aleatorizados que analizan los efectos de la aspiración de las secreciones subglóticas

\begin{tabular}{|c|c|c|c|c|c|}
\hline & Población & Casos/controles & Tipo aspiración & NAV incidencia & NAV (1.000 días VM) \\
\hline Mahul $^{3}$ & Polivalente & $70 / 75$ & Intermitente & $\begin{array}{l}\text { El } 13 \text { vs. el } 29 \% \\
p<0,05\end{array}$ & $\mathrm{NE}$ \\
\hline Smulders $^{6}$ & Polivalente & $75 / 75$ & Intermitente & $\begin{array}{l}\text { El } 4 \text { vs. el } 16 \% \\
p=0,014\end{array}$ & $\begin{array}{l}9,2 \text { vs. } 22,5 \\
p<0,001\end{array}$ \\
\hline Valles $^{7}$ & Polivalente & $76 / 77$ & Continua & $\begin{array}{l}\text { El } 18 \text { vs. el } 32 \% \\
\text { RR: } 1,76 ; \\
\text { IC: } 0,99-3,12\end{array}$ & $\begin{array}{l}19,9 \text { vs. } 39,6 \\
\text { RR: } 1,98 ; \\
\text { IC: } 1,03-3,82\end{array}$ \\
\hline Kollef ${ }^{21}$ & Cirugía cardiaca & $160 / 183$ & Continua & $\begin{array}{l}\text { El } 5 \text { vs. el } 8,2 \% \\
\text { RR: } 0,61 ; \\
\text { IC: } 0,27-1,4\end{array}$ & $\mathrm{NE}$ \\
\hline Bouza $^{22}$ & Cirugía cardiaca & $359 / 331$ & Continua & $\begin{array}{l}\text { El } 27 \text { vs. el } 47 \% \\
p=0,04^{a}\end{array}$ & $\begin{array}{l}\text { El } 31 \text { vs. el } 52 \% \\
p=0,03^{a}\end{array}$ \\
\hline
\end{tabular}

una mayor facilidad para adherirse y colonizar las células epiteliales traqueales que las bucales u orofaríngeas ${ }^{74-76}$. Efectivamente, en los trabajos de Mahul y Valles los autores encuentran que en una proporción considerable (el 40 y el $16,6 \%$, respectivamente) de las NAV producidas por BGN no fermentadores

hubo colonización previa de las secreciones subglóticas ${ }^{47,51}$. Si $P$. aeruginosa no coloniza ni se multiplica en el área subglótica, las medidas preventivas de NAV que afectan a las secreciones subglóticas no influirán en las neumonías causadas por este microorganismo.

Rello et al también evaluaron la aspiración continua de las secreciones subglóticas en una serie de 83 pacientes que recibieron VM y encontró que el fallo de la aspiración era un factor de riesgo independiente para NAV (RR: 5,29; IC del 95\%: $1,24-22,64)^{13}$.

Tal y como se ha comentado en el apartado anterior, Lorente et al demostraron cómo una combinación de neumotaponamiento de poliuretano y ASS continua disminuía drásticamente la aparición de $\mathrm{NAV}^{65}$.

En los estudios realizados en humanos no se ha detectado ningún efecto adverso de la ASS, incluso cuando establecer la seguridad del dispositivo era uno de los principales objetivos $^{66}$. Sin embrago, Berra et al realizaron un estudio en ovejas y detectaron una lesión difusa de la mucosa traqueal secundaria a la aspiración ${ }^{77}$.

Descontaminación digestiva selectiva (DDS). La descontaminación selectiva del tracto gastrointestinal, incluidas la aplicación antibiótica tópica en la orofaringe y el tracto gastrointestinal y la administración parenteral de antibióticos, es una medida ideada para la prevención de IN que aún está sumida en una gran controversia. Aunque numerosos trabajos han mostrado una reducción de la incidencia de NAV, los detractores del uso de DDS aducen que este resultado sólo se acompaña de una reducción de la mortalidad cuando los trabajos se analizan en forma de metaanálisis (se han publicado hasta 11$)^{78-80}$. Recientemente se han publicado 3 ensayos clínicos en los que se mostraba una disminución de la mortalidad pero nuevamente la aceptación general de la DDS fue limitada, por posibles defectos metodológicos, por ser los beneficios solo aplicables a un subgrupo de gravedad determinada por la Acute Physiology and Chronic Health Evaluation II (20-29 puntos) o por haberse realizado el estudio en un único centro ${ }^{81-83}$. Recientemente, Smet et al publicaron un ambicioso ensayo clínico multicéntrico que incluyó a $13 \mathrm{UCl}$ y 5.939 pacientes (1.990 asignados a grupo control, 1.904 asignados a descontaminación digestiva sin antibiótico parenteral y 2.045 asignados a descontaminación digestiva con antibiótico parenteral) ${ }^{84}$. Los resultados del trabajo mostraron una clara reducción de la mortalidad a los 28 días (una vez ajustado el resultado a las variables de confusión) tanto cuando se asociaba el antibiótico parenteral (el 13\% de reducción) como cuando se prescindía de este (el $11 \%$ de reducción). Se observó también una menor incidencia de bacteriemia en los pacientes descontaminados; curiosamente, los autores no aportan datos sobre el efecto en la aparición de NAV, ya que no formaba parte de los objetivos. Durante los 2 años en que se realizó el estudio, no se detectó un aumento de microorganismos multirresistentes. A pesar de los buenos resultados, hay quien sigue dudando sobre la generalización del uso de DDS en cuanto a sus posibles efectos en unidades con una elevada prevalencia de microorganismos multirresistentes. De hecho, se ha publicado un efecto deletéreo en unidades endémicas para SARM ${ }^{83,85}$ o para BGN multirresistentes ${ }^{86,87}$.

La creencia de algunos autores sobre la menor importancia del tracto gastrointestinal como fuente de microorganismos productores de $\mathrm{NAV}^{72}$, sobre todo cuando se mantiene la posición semiincorporada en todos los pacientes que reciben $\mathrm{VM}^{52}$, ha conducido hacia la aplicación de medidas descontaminantes más locales. La modulación de la flora bacteriana de la orofaringe mediante antibióticos tópicos ${ }^{88-91}$ o antisépti$\cos ^{92}$ ha resultado ser eficaz para la prevención de la NAV. Una nueva versión más sofisticada incluye la infusión continua de una solución antibiótica en el espacio subglótico mediante la luz dorsal del Hi-Lo Evac tube Mallinckrodt con aspiración orofaríngea horaria; 30 pacientes traumáticos se asignaron 
aleatorizadamente para recibir esta medida frente a 31 controles. Los autores describen una menor incidencia de NAV, una aparición más tardía de la NAV, una menor colonización del árbol bronquial y una reducción de la estancia en la $\mathrm{UCl}^{93}$. Esta idea parece estar corroborada por los resultados de Smet et al, ya que la adición del antibiótico parenteral apenas superaba los resultados obtenidos en ausencia de este ${ }^{84}$.

\section{Biofilm}

El biofilm es una compleja estructura formada por bacterias. Las bacterias adquieren una forma sesil hipometabólica y segregan, reguladas por unos genes específicos, una sustancia extracelular polimérica que las adhiere fuertemente entre sí y al sustrato elegido. La microbiología industrial ha estudiado ampliamente el biofilm, y sus hallazgos se han aplicado a la microbiología clínica94. La implicación del biofilm en las infecciones ocasionadas por la presencia de dispositivos artificiales endocorporales ha adquirido en los últimos años mucha importancia95-97.

El biofilm por su naturaleza viscosa y adherente no es fácilmente eliminable. Las bacterias embebidas en el biofilm quedan protegidas por este efecto mecánico, por la dificultad para la penetración de los antibióticos y por tener una mayor resistencia a los antibióticos. La resistencia antibiótica se debe al estado hipometabólico en el que se encuentran las bacterias pero también a una regulación genética muy próxima a la que modula la propia formación del biofilm ${ }^{98}$. El Pseudomonas spp. es el paradigma de bacteria que emplea el biofilm como parte de su arsenal patogénico; su presencia en el biofilm se ha relacionado con la NAV recurrente por este microorganismo ${ }^{99,100}$.

En 1986, Sottile et al fueron los primeros autores que observaron la presencia de biofilm en 25 TET utilizados en pacientes críticos; el $84 \%$ estaba totalmente tapizado y el $16 \%$ lo estaba parcialmente ${ }^{101}$. Posteriormente, Inglis et al encontraron biofilm en el $100 \%$ de los tubos analizados, incluidos 17 que se habían usado durante menos de $24 \mathrm{~h}$. El análisis microbiológico encontró bacterias en el $73 \%$ de los casos, fundamentalmente Pseudomonas spp. y Enterobacteriaceae. Estos autores simularon una VM in vitro con los tubos analizados y encontraron que las bacterias se proyectaban como mínimo $15 \mathrm{~cm}$ con una distribución elíptica $^{102}$.

Algunos autores analizaron la correlación entre las bacterias presentes en el biofilm y la etiología de la neumonía. Feldman et al observaron cómo, en 13 pacientes con neumonía, el estudio del biofilm de los TET retirados mostraba el mismo microorganismo en $8 \operatorname{casos}^{103}$. Adair et al observaron esta correlación en el $70 \%$ de los pacientes con neumonía y en ninguno de los pacientes que no habían desarrollado esta complicación ${ }^{104}$.

La implicación del biofilm en la patogenia de la NAV no está totalmente esclarecida, ya que podría tratarse de la fuente de la infección o ser un reservorio de bacterias potencialmente patógenas. Sin embargo, son numerosos los esfuerzos destinados a prevenir, eliminar o descontaminar el biofilm.

Prevención de la formación del biofilm. Jones et al demostraron in vitro cómo los tubos recubiertos de una solución antiséptica evitaban la formación del biofilm ${ }^{105,106}$. Estos resultados se corroboraron también in vivo y demostraron un menor grado de colonización en tubos impregnados con clorhexidina y plata $(\mathrm{p}<0,001)$; el análisis de los TET tras 5 días mostró una permanencia de un $45 \%$ del antiséptico $^{107}$.

El uso de biomateriales recubiertos de plata para la prevención de IN es una medida cuyo uso se está estudiando en relación con los catéteres endovasculares y urinarios; en estos últimos, su eficacia parece haberse demostrado ${ }^{108}$. Olson et al demostraron una menor colonización del TET, del árbol bronquial y del parénquima pulmonar en 5 perros con TET recubierto de plata frente a 6 controles tras la instilación de un inóculo de P. aeruginosa ${ }^{109}$.

Recientemente, Kollef et al demostraron en un ensayo clínico que incluyó a 9.417 pacientes de 54 centros, cómo el uso de TET recubiertos de plata lograba una reducción de la incidencia de NAV en los pacientes ventilados más de $24 \mathrm{~h}$ (finalmente, 968 pacientes en el grupo control y 964 con tubo de plata). La incidencia de NAV en el grupo control fue del $7,5 \%$, y en el grupo con tubo recubierto de plata la incidencia fue del 4,8\% $(p=0,03)$. Esta intervención probablemente requeriría un análisis de costes antes de su aplicación sistemática ${ }^{110}$.

Eliminación del biofilm. La eliminación mecánica completa del moco y el biofilm adheridos a la superficie interna del TET no es posible con las sondas de aspiración habituales. Recientemente se ha ideado un dispositivo capaz de realizar una limpieza mecánica del interior del TET, el mucus shaver. Se trata de una sonda con un balón inflable en su extremo que posee 2 anillos; una vez hinchado el balón en el interior del TET, los anillos obliteran el tubo y la extracción de la sonda logra la eliminación de toda la mucosidad adherida. Kolobow et al probaron este dispositivo en 6 ovejas que recibían VM frente a un grupo control formado por otras 2 ovejas. No observaron ningún problema técnico ni ningún efecto nocivo en la mecánica ventilatoria y cada uso del mucus shaver obtuvo una media de $0,35 \pm 0,29 \mathrm{~g}$ de moco. Tras $72 \mathrm{~h}$, el análisis por microscopia electrónica de los TET en el grupo control mostró una extensa formación de biofilm, mientras que este estaba ausente en el grupo estudio ${ }^{111}$.

El mismo grupo de autores repitió la experiencia en 12 ovejas intubadas con TET impregnado de una solución con plata-sulfadiacina; se trataron 6 ovejas cada $6 \mathrm{~h}$ con el mucus shaver. A diferencia del grupo control, en el grupo estudio no se encontró biofilm en el interior del TET tras $72 \mathrm{~h}$ de $\mathrm{VM}^{112}$.

Descontaminación del biofilm. Dado que son las bacterias las que forman el biofilm, la eliminación de estas supondría, a su vez, la desaparición del biofilm. En este sentido, el grupo de Adair et al estudió el efecto que la DDS (anfotericina B, tobramicina y polimixina) tenía sobre el biofilm; observaron una reducción en la colonización por enterobacterias pero una persistencia del biofilm formado por GP y Pseudomonas spp. Por otra parte, la concentración en el TET de los antibióticos usados fue errática y, con frecuencia, inferior a la concentración mínima inhibitoria necesaria, sobre todo en el caso de la tobramicina ${ }^{113,114}$.

Este mismo grupo de trabajo comparó el efecto que sobre el biofilm tenía el uso de cefotaxima parenteral, cefuroxima parenteral y gentamicina administrada en aerosol. Los TET 
de los 24 pacientes tratados con cefalosporinas parenterales desarrollaron biofilm en todos los casos, y en un $62,5 \%$ se encontraron microorganismos potencialmente patógenos. Los TET de los 12 pacientes tratados con gentamicina en aerosol desarrollaron biofilm en el $41,6 \%$ de los casos pero no se aisló ninguna bacteria considerada patógena. Los autores realizaron mediciones secuenciales de la concentración de los antibióticos en el interior del TET $2 \mathrm{~h}$ después de cada administración; las concentraciones de las cefalosporinas resultaron insuficientes para inhibir el crecimiento bacteriano, mientras que la gentamicina lograba mantener unas concentraciones correctas. Con respecto a la posible aparición de resistencias, tras un período de 3 años solo han aparecido algunas cepas de $S$. aureus resistente a la gentamicina ${ }^{115}$.

Actualmente, las actuaciones ensayadas con respecto al TET no han logrado contrarrestar totalmente los efectos deletéreos de este como factor favorecedor de NAV, pero los avances alcanzados indican un futuro más favorecedor.

\section{Conclusiones}

Considerar que las infecciones nosocomiales son errores evitables y conocer su incidencia son requisitos indispensables para su erradicación.

La prevención de las IN debe basarse en educación y preparación (técnica, pero también en seguridad) del personal sanitario, y asegurar un entorno de trabajo en equipo, en el que se apliquen las intervenciones de mayor evidencia y menor dificultad de implantación y coste. El seguimiento de protocolos generales y específicos, con la introducción de rutinas de comprobación que minimicen en un ambiente de mucha complejidad el riesgo del error, debe contar con la complicidad de todo el personal sanitario, incluidos los órganos directivos.

Por su número e impacto, los esfuerzos para prevenir la IN en el paciente crítico se centrarán en la NAV y la BRC.

\section{Bibliografía}

1. Institute of Medicine. To err is human: Building a safer health system. Kohn L, Corrigan J, Donaldson M, editores. Washington, DC: National Academy Press; 1999.

2. Rothschild JM, Landrigan CP, Cronin JW, Kaushal R, Lockley SW, Burdick E, et al. The Critical Care Safety Study: The incidence and nature of adverse events and serious medical errors in intensive care. Crit Care Med. 2005;33:1694-700.

3. Incidentes y eventos adversos en medicina intensiva. Seguridad y riesgo en el enfermo crítico. SYREC 2007. Informe, mayo 2009. Madrid: Ministerio de Sanidad y Política Social; 2009.

4. Leape LL. Reporting of adverse events. N Engl J Med. 2002; 347:1633-8.

5. Rosenthal MB. Nonpayment for Perfomance? N Engl J Med. 2007;357:1573-5.

6. Carlet J, Fabry J, Amalberti R, Degos L. The "Zero Risk" concept for Hospital-acquired infections: A risky Business. Clin Infect Dis. 2009;49:747-9.

7. Brown J, Doloresco F, Milotte JM. "Never events": Not every hospital-acquired infection is preventable. Clin Infect Dis. 2009;49:743-6.

8. Jarvis WR. The Lowbury Lecture. The United States approach to strategies in the battle against healthcare-associated infections, 2006: Transitioning from benchmarking to zero tolerance and clinician accountability. J Hosp Infect. 2007; 65:3-9.

9. Allegranzi B, Storr J, Dziekan G, Leotsakos A, Donaldson L, Pittet D. The First Global Patient Safety Challenge "Clean Care is Safer Care": From launch to current progress and achievements. J Hosp Infect. 2007;65:115-23.

10. Barbolla RE, Centrón D, Maimone S, Rospide F, Salgueira C, Altclas J, et al. Molecular epidemiology of Acinetobacter baumannii spread in an adult intensive care unit under an endemic setting. Am J Infect Control. 2008;36:444-52.

11. Dancer S, Coyne M, Speekenbrink A, Samavedam S, Kennedy J, Wallace P. MRSA acquisition in an intensive care unit. Am J Infect Control. 2006;34:10-7.

12. Goodman ER, Platt R, Bass R, Onderdonk AB, Yokoe DS, Huang $S S$, et al. Impact of an environmental cleaning intervention on the presence of methicillin- resistant Staphylococcus aureus and vancomycin-resistant enterococci on surfaces in intensive care unit rooms. Infect Control Hosp Epidemiol. 2008;29: 593-9.

13. Nulens $E$, Broex $E$, Ament A, Deurenberg RH, Smeets $E$, Scheres J, et al. Cost of the meticillin-resistant Staphylococcus aureus search and destroy policy in a Dutch university hospital. J Hosp Infect. 2008;68:301-7.

14. Haley RW, Bregman DA. The role of understaffing and overcrowding in recent outbreaks of staphylococcal infection in a neonatal special-care unit. J Infect Dis. 1982;145:875-85.

15. Fridkin SK, Pear SM, Williamson TH, Galgiani JN, Jarvis WR. The role of understaffing in central venous catheter-related bloodstream infections. Infect Control Hosp Epidemiol. 1996;17:150-8.

16. Clements A, Halton K, Graves N, Pettitt A, Morton A, Looke D, et al. Overcrowding and understaffing in modern health-care systems: Key determinants in Meticillin-Resistant Staphylococcus Aureus (MRSA) transmission. Lancet Infect Dis. 2008;8: 427-34.

17. Haley RW, Culver DH, White JW, Meade Morgan W, Emori TG, Munn VP, et al. The efficacy of infection surveillance and control programs in preventing nosocomial infections in US hospitals. Am J Epidemiol. 1985;121:182-205.

18. Warren DK, Zack JE, Mayfield JL, Chen A, Prentice D, Fraser VJ, et al. The effect of an education program on the incidence of central venous catheter-associated bloodstream infection in a medical ICU. Chest. 2004;126:1612-8.

19. Berenholtz SM, Pronovost PJ, Lipsett PA, Hobson D, Earsing K, Farley JE, et al. Eliminating catheter-related bloodstream infections in the intensive care unit. Crit Care Med. 2004: 2014.

20. Schweickert WD, Gehlbach BK, Pohlman AS, Hall JB, Kress JP. Daily interruption of sedative infusions and complications of critical illness in mechanically ventilated patients. Crit Care Med. 2004;32:1272-6.

21. Dries DJ, MCGonigal MD, Malian MS, Bor BJ, Sullivan C. Protocol-driven ventilator weaning reduces use of mechanical ventilation, rate of early reintubation, and ventilator-associated pneumonia. J Trauma. 2004;56:943-51.

22. Blamoun J, Alfakir M, Rella ME, Wojcik JM, Solis RA, Anees Khan $M$, et al. Efficacy of an expanded ventilator bundle for the reduction of ventilator-associated pneumonia in the medical intensive care unit. Am J Infect Control. 2009;37: $172-5$.

23. Institute for Healthcare Improvement. Getting started kit: prevent ventilator-associated pneumonia. How-to guide. 2008 [consultado 3/1/2009]. Disponible en: http://www.premie rinc.com/safety/topics/bundling/downloads/03-vap-how-toguide.pdf.

24. Bonello RS, Fletcher CE, Becker WK, Clutter KL, Arjes SL, Cook JJ, et al. An intensive care unit quality improvement collaborative in nine Department of Veterans Affairs hospitals: Reducing 
ventilator-associated pneumonia and catheter-related bloodstream infection rates. Jt Comm J Qual Patient Saf. 2008; 34:639-45.

25. Berwick DM, Calkins DR, McCannon CJ, Hackbarth AD. The 100.000 Lives Campaign: Setting a goal and a deadline for improving health care quality. JAMA. 2006;295:324-7.

26. Pronovost P, Needham D, Berenholtz S, Sinopoli D, Chu H, Cosgrove $\mathrm{S}$, et al. An intervention to decrease catheterrelated bloodstream infections in the ICU. N Engl J Med. 2006;355:2725-32.

27. Mermel LA. Prevention of intravascular catether-related infections. Ann Intern Med. 2000;132:391-402.

28. Hamilton HC, Foxcroff DR. Central venous access sites for the prevention of venous thrombosis, stenosis and infection in patients requiring long-term intravenous therapy. Cochrane Database Syst Rev. 2007:CD004084.

29. Merrer J, De Jonghe B, Golliot F, Lefrant JY, Raffy B, Barre E, et al. Complications of femoral and subclavian venous catheterization in critically ill patients: A randomized controlled trial. JAMA. 2001;286:700-7.

30. Lorente L, Jiménez A, Santana $M$, Iribarren JL, Jiménez JJ, Martín MM, et al. Microorganisms responsible for intravascular catheter-related bloodstream infection according to the catheter site. Crit Care Med. 2007;35:2424-7.

31. O'Grady NP, Alexander M, Dellinger EP, Gerberding JL, Heard SO, Maki DG, et al. Guidelines for the prevention of intravascular catheter-related infections. Centers for Disease Control and Prevention. MMWR Recomm Rep. 2002;51: $1-29$.

32. León C, Ariza J. Guías para el tratamiento de las infecciones relacionadas con catéteres intravasculares de corta permanencia en adultos: conferencia de consenso SEIMC-SEMICYUC. Enferm Infecc Microbiol Clin. 2004;22:92-101.

33. Suetens C, Lepape A, Palomar M, Hiesmayer M. Impact of risk adjustment on inter-countries comparisons of ICU infections indicators. ESCAIDE. 2007 Oct 18-20; Stockholm. Disponible en: http://helics.univ-lyon1.fr.

34. Álvarez-Lerma F, Palomar F, Olaechea P, Otal JJ, Insausti J, Cerdá E, Grupo de Estudio de Vigilancia de Infección Nosocomial en UCI. Estudio Nacional de Vigilancia de Infección Nosocomial en UCl. Informe de los años 2003-2005. Med Intensiva. 2007;31:6-17.

35. Raad IL, Hohn DG, Gilbreath BJ, Suleiman N, Hill LA, Bruso PA, et al. Prevention of central venous catheter-related infections by using maximal sterile barrier precautions during insertion. Infect Control Hosp Epidemiol. 1994;15(4 Pt 1): 231-8.

36. Mermel LA, McCormick SR, Maki DG. The pathogenesis and epidemiology of catheter-related infection with pulmonary artery Swan-Ganz catheters: A prospective study utilizing molecular subtyping. Am J Med. 1991;91:197S-205S.

37. Chajvakunapruk N, Veenstra DL, Lipsky BA, Saint S. Chlorhexidine compared with povidone-iodine solution for vascular catheter-site care: A meta-analysis. Ann Intern Med. 2002;136:792-801.

38. Garnacho-Montero J, Aldabó-Pallás T, Palomar-Martínez M, Vallés J, Almirante B, Garcés R. Risk factors and prognosis of catheter-related bloodstream infection in critically ill patients: A multicenter study. Intensive Care Med. 2008;34: 2185-93.

39. Yébenes JC, Vidaur L, Serra-Prat M, Sirvent JM, Batlle J, Motje M, et al. Prevention of catheter-related bloodstream infection in critically ill patients using a disinfectable, needle-free connector: A randomized controlled trial. Am J Infect Control. 2004;32:291-5.

40. Bouza E, Muñoz P, López-Rodríguez J, Pérez MJ, Rincón C, Martín Rabadan P. A needleless closed system device (CLAVE) protects from intravascular catheter tip and hub colonization:
A prospective randomized study. J Hosp Infect. 2003;54: 279-87.

41. Rupp ME, Sholtz LA, Jourdan DR, Marion ND, Tyner LK, Fey PD, et al. Outbreak of bloodstream infection temporally associated with the use of an intravascular needleless valve. Clin Infect Dis. 2007;44:1408-14.

42. Timsit JF, Schwebel C, Bouadma L, Geffroy A, GarrousteOrgeas $M$, Pease $S$, et al. Chlorhexidine-impregnated sponges and less frequent dressing changes for prevention of catheterrelated infections in critically ill adults: A randomized controlled trial. JAMA. 2009;301:1231-41.

43. Mermel LA. Prevention of central venous catheter-related infections: What works other than impregnated or coated catheters? J Hosp Infect. 2007:30-3.

44. Rupp ME, Lisco SJ, Lipsett PA, Perl TM, Keating K, Civetta JM, et al. Effect of a second-generation venous catheter impregnated with chlorhexidine and silver sulfadiazine on central catheter-related infections: a randomized, controlled trial. Ann Intern Med. 2005;143:570-80.

45. Guidelines for the management of adults with hospitalacquired, ventilator-associated, and healthcare-associated pneumonia. Am J Respir Crit Care Med. 2005;171:388-416.

46. Greene R, Thompson S, Jantsch HS, Teplik R, Cullen D, Green E, et al. Detection of pooled secretions above endotracheal-tube cuffs: Value of plain radiographs in sheep cadavers and patients. AJR. 1994;163:1333-7.

47. Mahul Ph, Auboyer C, Jospe R, Ros A, Guerin C, El Khouriz. Prevention of nosocomial pneumonia in intubated patients: Respective role of mechanical subglotic secretions drainage and stress ulcer prophylaxis. Intensive Care Med. 1992;18: 20-5.

48. Craven DE, Steger KA. Epidemiology of nosocomial pneumonia: New concepts on an old disease. Chest. 1995;108: $1 \mathrm{~S}-6 \mathrm{~S}$.

49. Torres A, Serra-Batlles J, Ros E, Piera C, Puig de la Bellacasa J, Cobos A, et al. Pulmonary aspiration of gastric contents in patients receiving mechanical ventilation: the effect of body position. Ann Intern Med. 1992;116:540-3.

50. Smulders K, Van der Hoeven H, Weers-Pothoff I, Vandenbroucke-Grauls C. A randomized clinical trial os intermittent subglottic secretion drainage in patients receiving mechanical ventilation. Chest. 2002;121:858-62.

51. Valles J, Artigas A, Rello J, Bonsoms N, Fontanals D, Blanch L, et al. Continuous aspiration of subglottic secretions in preventing ventilator-associated pneumonia. Ann Intern Med. 1995;122:179-86.

52. Draculovic MB, Torres A, Bauer TT, Nicolas JM, Noque S, Ferrer M. Supine body position as a risk factor for nosocomial pneumonia in mechanically ventilated patients: A randomised trial. Lancet. 1999;354:1851-8.

53. Van Nieuwenhoven CA, Vandenbroucke-Grauls C, van Tiel FH, Joore HC, Van Schijndel RJ, Van der Tweel I, et al. Feasibility and effects of the semi recumbent position to prevent ventilator-associated pneumonia: A randomized study. Crit Care Med. 2006;34:396-402.

54. Stauffer JL. Complications of translaryngeal intubation. En: Tobin MJ, editor. Principles and practice of mechanical ventilation. New York: McGraw-Hill Inc.; 1994. p. 711-48.

55. Estes RJ, Meduri GU. The pathogenesis of ventilator-associated pneumonia: I. Mechanism of bacterial translocation and airway inoculation. Intensive Care Med. 1995;21:365-83.

56. Knowlson GTG, Basset HFM. The pressures exerted on the trachea by endotracheal inflatable cuffs. $\mathrm{Br} \mathrm{J}$ Anaesth. 1970;42:834-7.

57. Stauffer JL, Olson DE, Petty TL. Complications and consequences of endotracheal intubation and tracheotomy: A prospective study of 150 critically ill adult patients. Am J Surg. 1981;70:65-75. 
58. Rello J, Sonora R, Jubert $P$, Artigas A, Rué $M$, Vallés J. Pneumonia inintubated patients: Role of respiratory airway care. Am J Respir Crit Care Med. 1996;154:111-5.

59. Seegobin RD, Van Hasselt GL. Aspiration beyond endotracheal cuffs. Can Anaesth Soc J. 1986;33:273-9.

60. Pavlin EG, Van Nimwegan D, Hornbein TF. Failure of a highcompliance low-pressure cuff to prevent aspiration. Anesthesiology. 1975;42:216-9.

61. Young PJ, Rollinson M, Downward G, Henderson S. Leakage of fluid past the tracheal tube cuf in a benchtop model. $\mathrm{Br} J$ Anaesth. 1997;78:557-62.

62. Blunt MC, Young PJ, Patil A, Haddock A. Gel lubrication of the tracheal tube cuff reduces pulmonary aspiration. Anesthesiology. 2001;95:377-81.

63. Young PJ, Burchett K, Harvey I, Blust MC. The prevention of pulmonary aspiration with control of tracheal wall pressure using a silicone cuff. Anaesth Intensive care. 2000;28:660-5.

64. Young PJ, Pakeerathan S, Blunt MC, Subramanya S. A lowvolume, low-pressure tracheal tube cuff reduces pulmonary aspiration. Crit Care Med. 2006;34:632-9.

65. Lorente L, Lecuona M, Jiménez A, Mora ML, Sierra A. Influence of an endotracheal tube with polyurethane cuff and subglottic secretion drainage on pneumonia. Am J Respir Crit Care Med. 2007; 176:1079-83.

66. Kollef MH, Skubas NJ, Sundt TM. A randomized clinical trial of continuous aspiration of subglottic secretions in cardiac surgery patients. Chest. 1999;116:1339-46.

67. Bouza E, Pérez MJ, Muñoz P, Rincón C, Barrio JM, Hortal J. Continuous aspiration of subglottic secretions in the prevention of ventilator-associated pneumonia in the postoperative period of major heart surgery. Chest. 2008;134:938-46.

68. Onofrio JM, Toews GB, Lipscomb MF, Pierce AK. Granulocytealveolar-macrophage interaction in the pulmonary clearance of Staphylococcus aureus. Am Rev Respir Dis. 1983;127: 335-41.

69. Toews GB, Gross GN, Pierce AK. The relationshipn of inoculum size to lung bacterial clearance and phagocytic response in mice. Am Rev Respir Dis. 1979;120:559-66.

70. De la Torre FJ, Pont T, Ferrer A, Rosselló J, Palomar M, Planas M. Pattern of tracheal colonization during mechanical ventilation. Am J Respir Crit Care Med. 1995;152: 1028-33.

71. Ewig S, Torres A, El-Elbiary, Fabregas N, Hernández C, González J, et al. Bacterial colonization patterns in mechanically ventilated patients with traumatic and medical head injury. Incidence, risk factors, and association with ventilator-associated pneumonia. Am J Respir Crit Care Med. 1999;159:188-98

72. Bonten MJ, Gaillard CA, Van Tiel FH, Smeets HG, van der Geest S, Stobberingh EE. The stomach is not a source for colonization of the upper respiratory tract and pneumonia in ICU patients. Chest. 1994;105:878-84.

73. Cardeñosa JA, Solé-Violán J, Bordes A, Noguera J, Arroyo J, Saavedra $P$, et al. Role of different routes of tracheal colonization in the development of penumonia in patients receiving mechanical ventilation. Chest. 1999;116:462-70.

74. Niederman MS, Mantovani R, Schoch P, Pappas J, Fein AM. Patterns and routes of tracheobronquial colonization in mechanically ventilated patients. The role of nutritional status in the colonization of the lower airway by Pseudomonas species. Chest. 1989;95:155-61.

75. Niederman MS. Gram-negative colonization of the respiratory tract: pathogenesis and clinical consequences. Semin Respir Infect. 1990;5:173-84.

76. Niederman MS, Rafferty TD, Sasaki CT, Merrill V, Mathay R, Reynolds $\mathrm{H}$. Comparison of bacterial adherence to ciliated and squamous epithilial cells obtained from the human rspiratory tract. Am Rev Respir Dis. 1983;127:85-90.
77. Berra L, De Marchi L, Panigada M, Yu ZX, Bacarelli A, Kolovow T. Evaluation of continuous aspiration of subglottic secretion in a in vivo study. Crit Care Med. 2004;32:2071-8.

78. Smet $A M$, Bonten MJ. Selective decontamination of the digestive tract. Curr Opin Infect Dis. 2008;21:179-83.

79. García-Hierro P, De la Cal MA, Van Saene HK, Silvestri L. Un nuevo ensayo clínico con descontaminación digestiva selectiva. Med Intensiva. 2009;33:297-300.

80. Bonten MJM. Selective digestive tract decontamination. Hill it prevent infection with multidrug resistant gram-negative pathogens but still be aplicable in institutions where meticillin-resistant Staphylococcus aureus and vancomycinresistant Enterococci are endemic? CID. 2006;43:S70-4.

81. De Jonge E, Schultz M, Spanjaard L, Bossuyt P, Vroom M, Dankert J. Effects of selective decontamination of the digestive tract on mortality and acquisition of resistant bacteria in intensive care: A randomized controlled trial. Lancet. 2003;362:1011-6.

82. Krueger WA, Lenhart FP, Neeser G, Ruckdeschel G, Schreckhase $\mathrm{H}$, Eissner $\mathrm{H}$. Influence of combined intravenous and topical antibiotic prophylaxis on the incidence of infections, organ disfunctions, and mortality in critically ill surgical patients: A prospective, stratified, randomized, double-blind, placebo-controlled clinical trial. Am J Respir Crit Care Med. 2002;166:1029-37.

83. De la Cal MA, Cerdá E, García-Hierro P, van Saene R, Gómez D, Negro E. Survival benefit in critically ill burned patients receiving selective decontamination of the digestive tract. A randomized, placebo-controlled, double-blind trial. Ann Surg. 2005;241:424-30.

84. Smet AM, Kluytmans JA, Cooper BS, Mascini EM, Benus RF, van der Werf TS, et al. Decontamination of the digestive tract and oropharynx in ICU patients. N Engl J Med. 2009;360:20-31.

85. Lingnau W, Berger J, Javorsky F, Fille M, Allerbierger F, Benzer H. Changing bacterial ecology during a five-year period of selective intestinal decontamination. J Hosp Infect. 1998;39: 195-206.

86. Verwaest C, Verhaegen J, Ferdinande P, Schetz M, van der Berghe $G$, Verloist L, et al. Randomized, controlled trial of selective decontamination in 600 mechanically ventilated patients in a multidisciplinary intensive care unit. Crit Care Med. 1997;25:63-71.

87. Hammond JMJ, Potgieter PD, Saunders GL, Forder AA. Doubleblind study of selective decontamination of the digestive tract in intensive care. Lancet. 1992;340:5-9.

88. Bergmans DC, Bonten MJ, Gaillard CA, Paling JC, van der Geest S, van Tiel FH, et al. Prevention of ventilatorassociated pneumonia by oral decontamination: A prospective, randomized, double-blind, placebo-controlled study. Am J Respir Crit Care Med. 2001;164:382-8.

89. Abele-Horn M, Bauber A, Baurnfeind A, Russwurm W, SeyfarthMetzger I, Gleich P, et al. Decrease in nosocomial pneumonia in ventilated patients by selective oropharyngeal decontamination. Intensive Care Med. 1996;23:187-95.

90. Pugin J, Auckenthaler R, Lew DP, Suter P. Oropharyngeal decontamination decreases incidence of ventilator-associated pneumonia: A randomized, placebo-controlled, double-blind clinical trial. JAMA. 1991;265:2704-10.

91. Rodríguez-Roldán JM, Altuna-Cuesta A, López A, Carrillo A, Garcia J, León J, et al. Prevention of nosocomial lung infection in ventilated patients: Use of an antimicrobial pharyngeal nonabsorbable paste. Crit Care Med. 1990;18:1239-42.

92. DeRiso 2nd AJ, Ladowski JS, Dillon TA, Justice J, Paterson A. Chlorexidine gluconate $0.12 \%$ oral rinse reduces the incidente of total nosocomial respiratory infection and nonprophylactic systemic antibiotic use in patients undergoing cardiac surgery. Chest. 1996;109:1556-61. 
93. Pneumatikos I, Koulouras V, Nathanail C, Goe D, Nakos G. Selective decontamination of subglotic area in mechanically ventilated patients with multiple trauma. Intensive Care Med. 2002;28:432-7.

94. Donlan RM, Costerton W. Biofilms: Survival mechanisms of clinically relevant microorganisms. Clin Microbiol Rev. 2002;15:167-93.

95. Donlan RM. Biofilm formation: A clinically relevant microbiological proces. Clin Infect Dis. 2001;33:1387-92.

96. Gilbert P, McBain AJ. Biofilms: Their impact on health and their reclacitrance toward biocides. Am J Infect Control. 2001;29:252-5.

97. Donlan RM. Biofilms and device-associated infections. Emerg Infect Dis. 2001;7:277-81.

98. Stewarts PS, Costernon JW. Antibiotic resistance of bacteria in biofilms. Lancet. 2001;358:135-8.

99. Prince AS. Biofilms, antimicrobial resistence, and airway infection. N Engl J Med. 2002;347:1110-1.

100. Cai S, Zhang J, Qian G. Correlation of endotracheal tube biofilm and recurrent ventilator-associated pneumonia with Pseudomonas aeruginosa. Zhonghua Jie HeHe Hu Xi Za Zhi. 2001;24:339-41.

101. Sottile FD, Marrie TJ, Prough DS, Hobgood C, Gower D, Webb L. Nosocomial pulmonary infection: possible etiologic significance of bacterial adhesion to endotracheal tubes. Crit Care Med. 1986;14:267-70.

102. Inglis TJJ, Millar MR, Jones G, Robinson D. Tracheal tube biofilm as a source of bacterial colonization of the lung. J Clin Microbiol. 1989;27:2014-8.

103. Feldman C, Kassel M, Cantrell J, Kaka S, Morar R, Goolam M. The presence and sequence of endotracheal tube colonization in patients undergoing mechanical ventilation. Eur Respir J. 1999;13:546-51.

104. Adair C, Gorman SP, Feron BM, Byers M, Jones D, Goldsmith C, et al. Implications of endotracheal tube biofilm for ventilatorassociated pneumonia. Intensive Care Med. 1999;25:1072-6.

105. Jones DS, MvGovern JG, Woolfson AD. Physicochemical characterization of hexetidine-impregnated ebdotracheal tube poly (vinyl chloride) and resistance to adherence of respiratory bacterial pathogens. Pharm Res. 2002;19:818-24.
106. Jones DS, McMeel S, Adair CG, Gorman SP. Characterization and evaluation of novel surfactant bacterial anti-adherent coatings for endotracheal tubes designed for the prevention of ventilator-associated pneumonia. J Pharm Pharmacol. 2003; 55:43-52.

107. Pacheco-Fowler V, Gaonkar T, Wyer PC, Modak S. Antiseptic impregnated endotracheal tubes for the prevention of bacterial colonization. J Hosp Infect. 2004;57:170-4.

108. Stickler DJ. Biomaterials to prevent nosocomial infections: Is silver the gold standard? Curr Opin Infect Dis. 2000;13: 389-93.

109. Olson ME, Harmon BG, Kollef MH. Silver-coated endotracheal tubes associated with reduced bacterial burden in the lungs of mechanically ventilated dogs. Chest. 2002;121: 863-70.

110. Kollef MH, Afessa B, Anzueto A, Veremakis C, Kerr KM, Margolis $\mathrm{BD}$, et al. Silver-coated endotracheal tubes and incidence of ventilator-associated pneumonia: The NASCENT randomized trial. JAMA. 2008;300:805-13.

111. Kolobow T, Berra L, Li Bassi G, Curzo F. Novel system for complete removal of secretions within the endotracheal tube. The mucus shaver. Anesthesiology. 2005;102:1063-5.

112. Berra L, Curto F, Li Bassi G, Laquerriere P, Baccarelli A, Kolobow T. Antibacterial-coated tracheal tubes cleaned with the Mucus shaver. A novel method to retain long-term bactericidal activity of coated tracheal tubes. Intensive Care Med. 2006;32:888-93.

113. Gorman SP, Adair CG, O'Neill FB, Goldsmith C, Webb H. Influence of selective decontamination of the digestive tract on microbial biofilm formation on endotracheal tubes from artifically ventilated patients. Eur J Clin Microbiol Infect Dis. 1993;12:9-17.

114. Adair CG, Gorman SP, O'Neill FB, McClurg B, Goldsmith E, Webb $C$. Selective decontamination of the digestive tract (SDD) does not prevent the formation of microbial biofilms on endotracheal tubes. J Antimicrob Chemother. 1993;31: 689-97.

115. Adair CG, Gorman SP, Byers LM, Feron B, Crowe M, Webb H. Erradication of endotracheal tube biofilm by nebulised gentamicin. Intensive Care Med. 2002;28:426-31. 